\title{
Bevacizumab in the treatment of five patients with breast cancer and brain metastases: Japan Breast Cancer Research Network-07 trial
}

This article was published in the following Dove Press journal:

OncoTargets and Therapy

14 September 2012

Number of times this article has been viewed

\author{
Daigo Yamamoto ${ }^{1,3}$ \\ Satoru Iwase ${ }^{2}$ \\ Yu Tsubota' \\ Noriko Sueoka' \\ Chizuko Yamamoto ${ }^{3}$ \\ Kaoru Kitamura ${ }^{4}$ \\ Hiroki Odagiri ${ }^{5}$ \\ Yoshinori Nagumo 6 \\ 'Department of Surgery, Kansai \\ Medical University, Hirakata, Osaka, \\ ${ }^{2}$ Department of Palliative Medicine, \\ University of Tokyo Hospital, Tokyo, \\ ${ }^{3}$ Department of Internal Medicine, \\ Seiko Hospital, Neyagawa, Osaka, \\ ${ }^{4}$ Breast Unit, Nagumo Clinic, Fukuoka, \\ ${ }^{5}$ Department of Surgery, Hirosaki \\ National Hospital, Hirosaki, ${ }^{6}$ Breast \\ Unit, Nagumo Clinic, Tokyo, Japan
}

Correspondence: Daigo Yamamoto Department of Surgery,

Kansai Medical University, Hirakata,

Osaka 570-8507, Japan

Tel +81728040101

Fax +81728040170

Email yamamotd@hirakata.ac.jp
Background: Brain metastases from breast cancer occur in $20 \%-40 \%$ of patients, and the frequency has increased over time. New radiosensitizers and cytotoxic or cytostatic agents, and innovative techniques of drug delivery are still under investigation.

Methods: Five patients with brain metastases who did not respond to whole-brain radiotherapy and then received bevacizumab combined with paclitaxel were identified using our database of records between 2011 and 2012. The clinicopathological data and outcomes for these patients were then reviewed.

Results: The median time to disease progression was 86 days. Of five patients, two (40\%) achieved a partial response, two had stable disease, and one had progressive disease. In addition, one patient with brain metastases had ptosis and diplopia due to metastases of the right extraocular muscles. However, not only the brain metastases, but also the ptosis and diplopia began to disappear after 1 month of treatment. The most common treatment-related adverse events (all grades) were hypertension (60\%), neuropathy (40\%), and proteinuria (20\%). No grade 3 toxicity was seen. No intracranial hemorrhage was observed.

Conclusion: We present five patients with breast cancer and brain metastases, with benefits from systemic chemotherapy when combined with bevacizumab.

Keywords: brain, bevacizumab, metastatic breast cancer

\section{Introduction}

Breast carcinoma is the most frequent neoplasia in the US, Europe, and even Japan. ${ }^{1}$ Approximately $40 \%-45 \%$ of all patients with breast cancer will develop metastasis, and the mean survival time from the diagnosis of recurrence for these patients is 18-30 months. $^{2}$ Therefore, treatment of patients with metastatic breast cancer aims to prolong survival while relieving symptoms and maintaining a good quality of life. ${ }^{1-4}$ Brain metastases from breast cancer occur in $20 \%-40 \%$ of patients, and the frequency has increased over time. As a treatment, the combination of surgery and whole-brain radiotherapy is well known and useful, but is still limited. New radiosensitizers and cytotoxic or cytostatic agents and innovative techniques of drug delivery are being investigated. ${ }^{5}$

Bevacizumab has selective activity against the vascular endothelial growth factor (VEGF)-A ligand and has proven to be efficacious when combined with paclitaxel. ${ }^{6}$ It has been well documented that tumor blood vessels show increased vascular permeability and interstitial fluid pressure, decreased pericyte coverage, and increased occurrence of tumor hypoxia, further upregulating VEGF production. Therefore, inhibition of 
VEGF by bevacizumab will not only affect endothelial cells but also the tumor vasculature, suppressing new blood vessel growth and the existing vasculature.

Cautious use of bevacizumab has been recommended in patients at risk of bleeding and uncontrolled hypertension, as well as in patients with a history of arterial thrombotic events. Patients with central nervous system metastases have until recently been routinely excluded from bevacizumab trials, following a single case in 1997 of a 29-year-old patient with hepatocellular carcinoma who experienced a fatal cerebral hemorrhage from a previously undiagnosed brain metastasis in a Phase I study of bevacizumab. ${ }^{7}$ However, bevacizumab recently gained accelerated approval from the US Food and Drug Administration for progressive primary brain tumors, with a low rate (approximately 3\%) of intratumoral hemorrhage. ${ }^{8,9}$ More recent studies showed that bevacizumab is safe in patients with brain metastases. ${ }^{10-13}$ We present here the efficacy and side effects of bevacizumab for patients with breast cancer and brain metastases.

\section{Materials and methods}

From the Japan Breast Cancer Research Network database, we retrospectively identified five patients treated with bevacizumab-containing chemotherapy regimens for active central nervous system metastases. All patients had recurrent tumors after receiving radiation therapy. All patients received bevacizumab at a dose of $10 \mathrm{mg} / \mathrm{kg}$ by intravenous infusion every 2 weeks with concomitant paclitaxel. Paclitaxel $80 \mathrm{mg} / \mathrm{m}^{2}$ was administered intravenously on days 1,8 , and 15 every 4 weeks. Dose reductions of paclitaxel from 80 to $60 \mathrm{mg} / \mathrm{m}^{2}$ were performed as described previously. ${ }^{14}$ Tumor response was determined by comparing measurements from consecutive magnetic resonance imaging (MRI) scans, as described elsewhere. ${ }^{8}$ In brief, progressive disease was deemed to be present if a new lesion had occurred, if the MRI showed a $>25 \%$ increase in fluid attenuated inversion recovery (FLAIR) or contrast-enhanced volume, or if the MRI scan showed an increase in tumor volume; partial response was defined as a $>25 \%$ decrease in the enhanced lesion and FLAIR; and a complete response was defined as no detectable contrast enhancement and stable or improved FLAIR signal. Physical examination findings, tumor characteristics, number of treatment cycles, chemotherapyrelated toxicities, and symptom severity were recorded every week. Toxicity was graded using the National Cancer Institute Common Terminology Criteria for Adverse Events version 3.0 .

\section{Results}

\section{Patient characteristics}

The characteristics of the study population are presented in Table 1. The median age was 60 (range 39-71) years. Eastern Cooperative Oncology Group performance status was $<3$. All patients were pretreated with whole-brain radiotherapy. The median number of metastatic sites was three (range 1-5).

\section{Efficacy}

The patients were evaluable for response and toxicity. Of the five patients, two (40\%) achieved a partial response, two had stable disease, and one had progressive disease. Representative data are shown in Figures 1-4. In addition, one patient with brain metastases had ptosis and diplopia due to metastases of the right extraocular muscles (Figures 4 and 5). However, not only the brain metastases but also the ptosis and diplopia began to disappear after 1 month of treatment (Figure 5). Median time to disease progression was 86 (range 30-135) days. Two patients $(40 \%)$ were still alive at the last follow-up.

\section{Safety}

The most common treatment-related adverse events were grade $1 / 2$ in intensity. Common toxicities were

Table I Patient characteristics

\begin{tabular}{ll}
\hline Patients $(\mathbf{n}=\mathbf{5})$ & \\
\hline Age, years, median (range) & 60 (39-7I) \\
Performance status & 0 \\
0 & 2 \\
$\mathrm{I}$ & 2 \\
2 & \\
Hormone status & 3 \\
+ & 2 \\
- & \\
HER2 status & 1 \\
+ & 4 \\
- & \\
Menopausal status & 2 \\
Pre & 3 \\
Post & \\
Type of prior chemotherapy & 1 \\
Trastuzumab & 4 \\
Taxane & $\mathrm{I}$ \\
Anthracycline/taxane & \\
Whole-brain radiotherapy & 5 \\
+ & 0 \\
- & $3(I-5)$ \\
Median number of brain metastatic sites (range) & 2 \\
Metastatic sites involved & 2 \\
Bone/soft tissue & \\
Visceral & \\
\hline
\end{tabular}



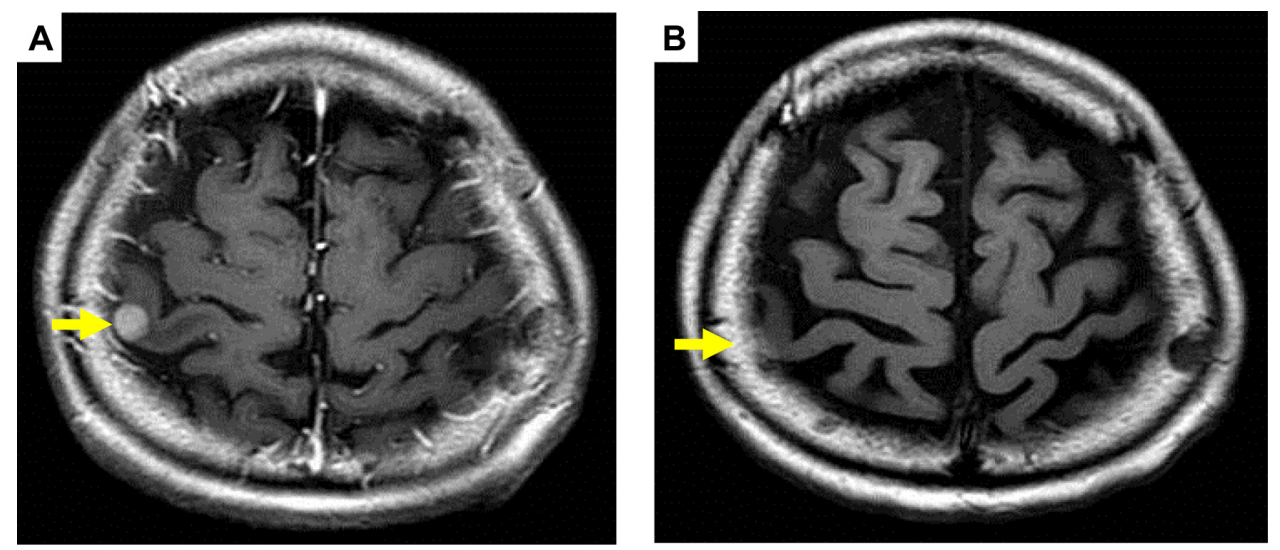

Figure I Pre (A) and post (B) treatment brain magnetic resonance imaging of metastatic tumor showing a partial response (arrows).
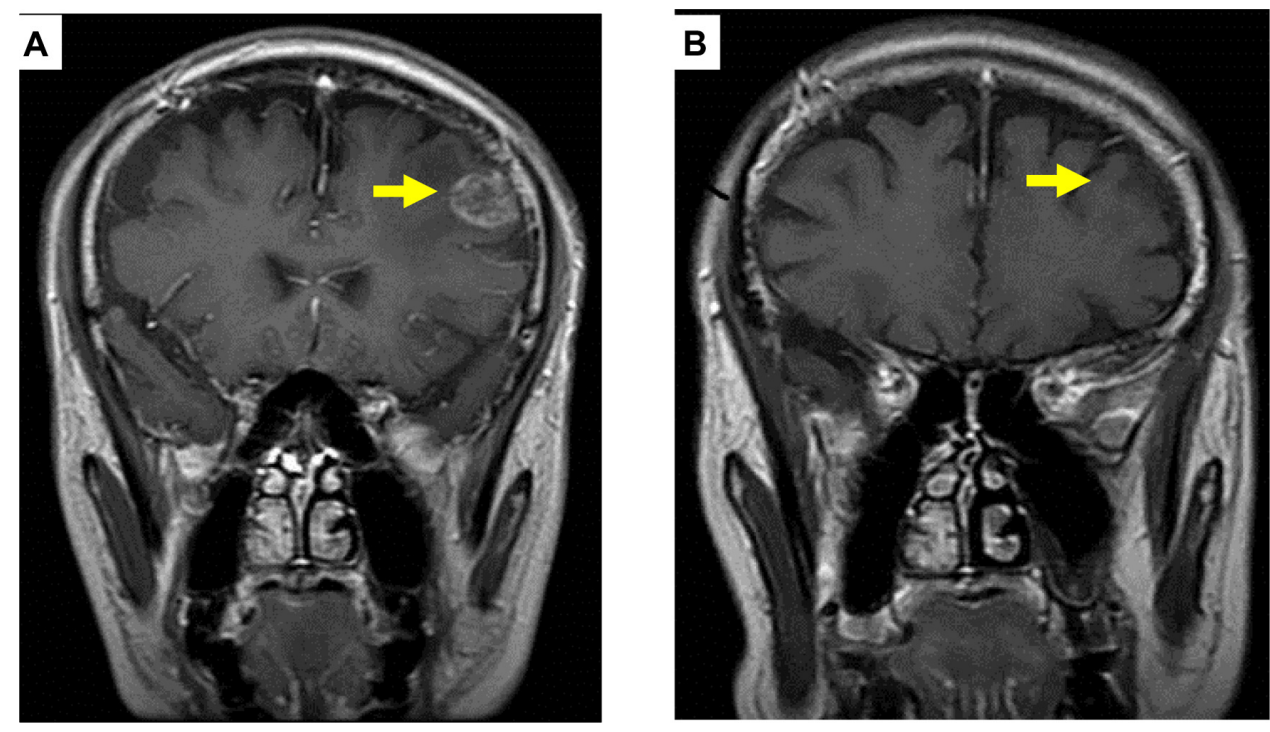

Figure 2 Pre (A) and post (B) treatment brain magnetic resonance imaging of metastatic tumor showing a complete response (arrows).
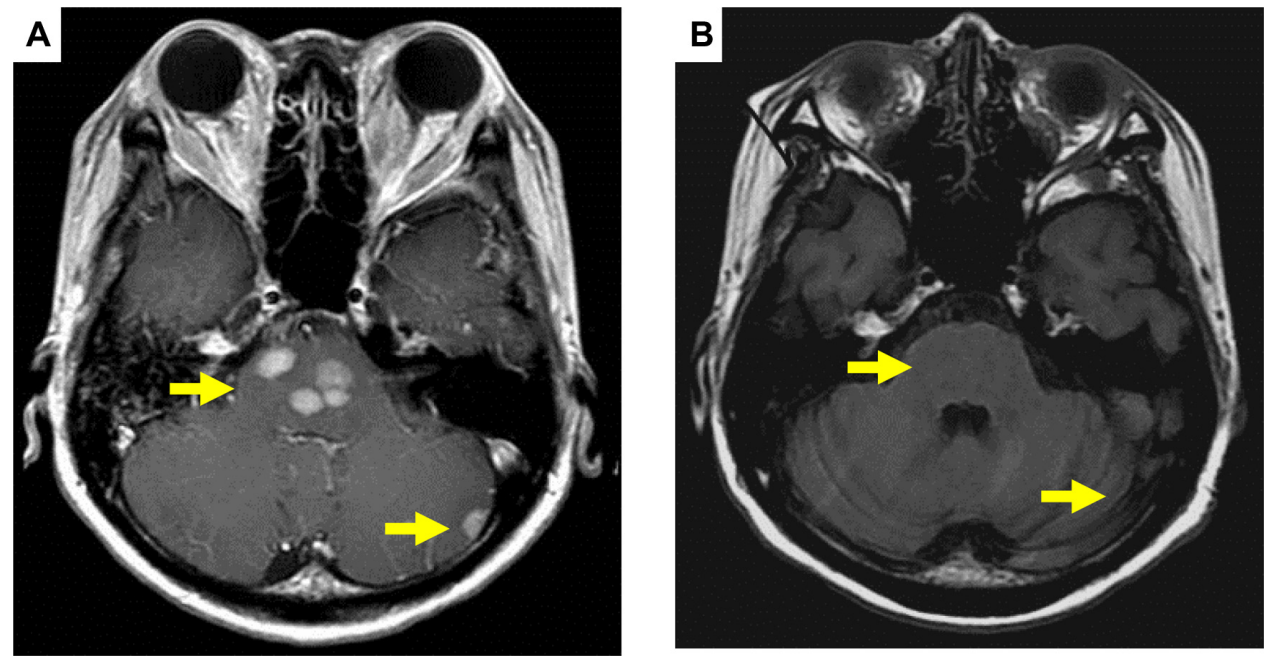

Figure 3 Pre $(\mathbf{A})$ and post $(\mathbf{B})$ treatment brain magnetic resonance imaging of metastatic tumor showing a partial response (arrows). 

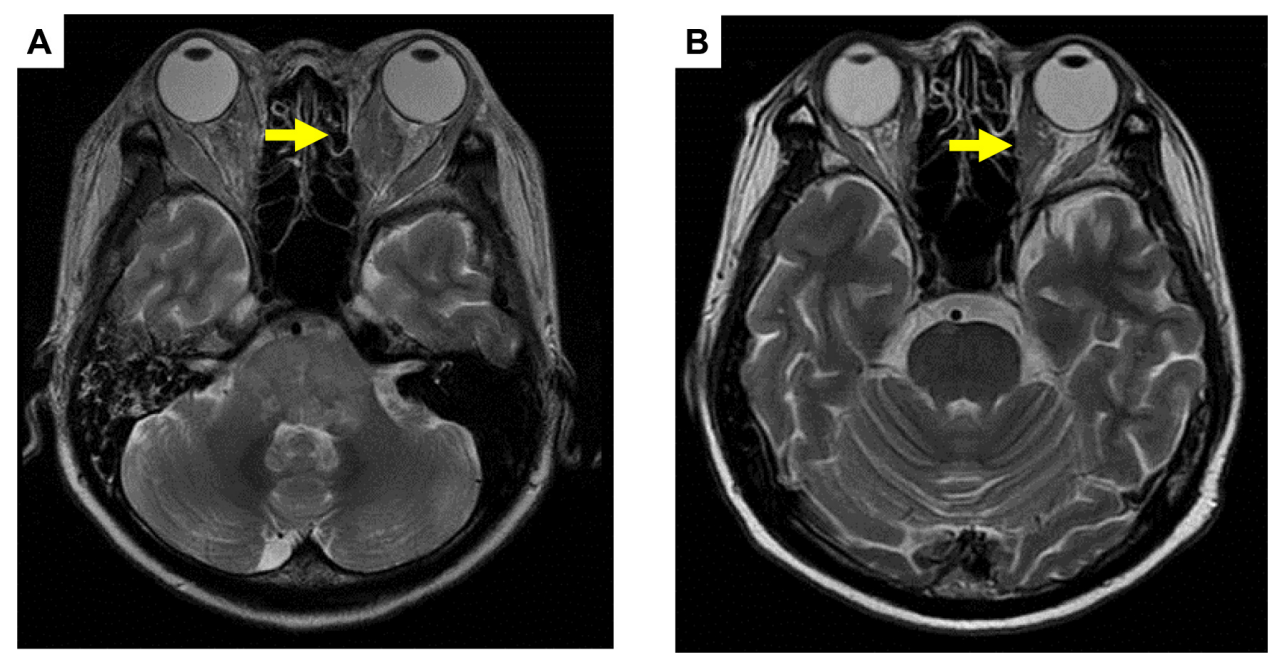

Figure 4 Pre $(\mathbf{A})$ and post $(\mathbf{B})$ treatment brain magnetic resonance imaging of metastases of right extraocular muscles showing a partial response (arrows).

grade 1 hypertension (60\%), grade 1 neuropathy $(40 \%)$, and proteinuria $(20 \%)$. No grade 3 toxicity was seen. No intracranial hemorrhage was observed.

\section{Discussion}

The results of this multicenter retrospective study suggest that bevacizumab combined with a taxane is highly active and well tolerated by women with breast cancer and brain metastases who have failed whole-brain radiotherapy. Bevacizumab combined with a taxane yielded a $40 \%$ response rate. The median time to disease progression was 86 days. The results presented here are similar to those reported elsewhere. ${ }^{12}$ The exact mechanism of bevacizumab in brain parenchymal disease is unknown; whether it is a result of direct effects on the tumor vasculature and/or the blood-brain barrier itself is unclear. Most drugs fail to enter the central nervous system because of the blood-brain barrier. This restriction particularly affects drugs that are not substrates for active transport into the central nervous system, hydrophilic molecules larger than $500 \mathrm{Da}$, and high molecular weight therapeutic modalities, such as monoclonal antibodies, antisense oligonucleotides, viral vectors, stem cells, and nanoparticles. ${ }^{15}$ However, some studies have shown that VEGF may provide new opportunities for manipulating the permeability of the blood-brain barrier in vivo. ${ }^{16,17}$ Further, previous studies in glioma models have demonstrated a fine balance between VEGF and angiopoietin-2, a proapoptotic factor in angiogenesis. ${ }^{18}$ It has been noted that the blood-brain barrier is abnormal with tumors $>0.5 \mathrm{~mm}$, and might affect the integrity of astrocytes and the endothelial cells of the blood-brain barrier. ${ }^{19}$ Larger tumors result in an increased risk of ischemia, further disrupting the blood-brain barrier. ${ }^{20}$ Additional studies are in progress to evaluate the role of bevacizumab in combination with chemotherapy in previously treated brain metastases originating from non-small-cell lung cancer and also in reducing central nervous system side effects after radiotherapy in patients with primary brain, melanoma, and head and neck cancer. ${ }^{21,22}$ In the present study, bevacizumab and paclitaxel suppressed brain metastasis. Therefore, theoretically, there is a possibility that bevacizumab might cross the blood-brain barrier and penetrate brain tumors in sufficient concentrations to synergize with anticancer drugs.
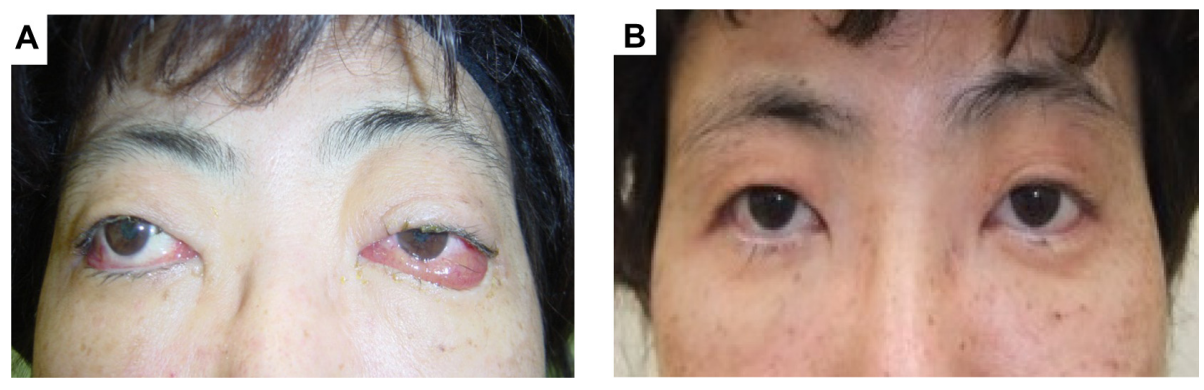

Figure 5 Facial features pre (A) and post (B) treatment.

Note: Ptosis and diplopia begin to disappear after I month of the treatment. 
In the current study, the majority of adverse events were mild to moderate in intensity, and confirm the results of previous studies in similar patient populations. ${ }^{11-13}$ Acute toxicities were quite mild and manageable. Hypertension and proteinuria were common, and neuropathy was managed with modification of the paclitaxel dose. Further, intracranial hemorrhage was not observed. The limitations of the present study include its retrospective nature and the small number of patients included. Nonetheless, the finding that bevacizumab has significant activity against breast cancer with brain metastasis is important.

\section{Acknowledgment}

The authors are indebted to Mr Ito and Mr Yamaga from Chugai Pharmaceutical Co, Ltd.

\section{Disclosure}

The authors report no conflicts of interest in this work.

\section{References}

1. Shin HR, Boniol M, Joubert C, et al. Secular trends in breast cancer mortality in five East Asian populations: Hong Kong, Japan, Korea, Singapore and Taiwan. Cancer Sci. 2010;101:1141-1246.

2. Perez EA. Current management of metastatic breast cancer. Semin Oncol. 1999;26:1-10.

3. Kawaguchi T, Iwase S, Takeuchi H, et al. Chemotherapy with low-dose capecitabine as palliative treatment in a patient with metastatic breast cancer: a case report. Cases J. 2009;24:9081.

4. Hortobagyi GN. Treatment of breast cancer. N Engl J Med. 1998;339: 974-984.

5. Tsao MN, Lloyd N, Wong RK, et al. Whole brain radiotherapy for the treatment of newly diagnosed multiple brain metastases. Cochrane Database Syst Rev. 2012;4:CD003869.

6. Croom KF, Dhillon S. Bevacizumab: a review of its use in combination with paclitaxel or capecitabine as first-line therapy for HER2-negative metastatic breast cancer. Drugs. 2011;71:2213-2229.

7. Gordon MS, Margolin K, Talpaz M. Phase I safety and pharmacokinetic study of recombinant human anti-vascular endothelial growth factor in patients with advanced cancer. J Clin Oncol. 2001;19:843-850.
8. Friedman HS, Prados MD, Wen PY, et al. Bevacizumab alone and in combination with irinotecan in recurrent glioblastoma. J Clin Oncol. 2009;27:4733-4740.

9. Agha CA, Ibrahim S, Hassan A, Elias DA, Fathallah-Shaykh HM. Bevacizumab is active as a single agent against recurrent malignant gliomas. Anticancer Res. 2010;30:609-611.

10. Socinski MA, Langer CJ, Huang JE, et al. Safety of bevacizumab in patients with non-small-cell lung cancer and brain metastases. J Clin Oncol. 2009;27:5255-5261.

11. Besse B, Lasserre SF, Compton P, Huang J, Augustus S, Rohr UP. Bevacizumab safety in patients with central nervous system metastases. Clin Cancer Res. 2010;16:269-278.

12. Labidi SI, Bachelot T, Ray-Coquard I, et al. Bevacizumab and paclitaxel for breast cancer patients with central nervous system metastases: a case series. Clin Breast Cancer. 2009;9:118-121.

13. Bhaskara A, Eng C. Bevacizumab in the treatment of a patient with metastatic colorectal carcinoma with brain metastases. Clin Colorectal Cancer. 2008;7:65-68.

14. Yamamoto D, Iwase S, Yoshida $\mathrm{H}$, et al. Efficacy of meloxicam in combination with preoperative chemotherapy for breast cancer-Japan Breast Cancer Research Network (JBCRN) 02-1 Trial. Anticancer Res. 2011;31:3567-3572.

15. De Boer AG, Gaillard PJ. Strategies to improve drug delivery across the blood-brain barrier. Clin Pharmacokinet. 2007;46:553-576.

16. Ferrara N, Gerber HP, LeCouter J. The biology of VEGF and its receptors. Nat Med. 2003;9:669-676.

17. Ay I, Francis JW, Brown RH. VEGF increases blood-brain barrier permeability to Evans blue dye and tetanus toxin fragment $\mathrm{C}$ but not adeno-associated virus in ALS mice. Brain Res. 2008;1234:198-205.

18. Zadeh G, Guha A. Molecular regulators of angiogenesis in the developing nervous system and adult brain tumors. Int J Oncol. 2003;23: $557-565$.

19. Gerstner ER, Fine RL. Increased permeability of the blood-brain barrier to chemotherapy in metastatic brain tumors: establishing a treatment paradigm. J Clin Oncol. 2007;25:2306-2312.

20. Dietrich WD, Busto R, Halley M. The importance of brain temperature in alterations of the blood-brain barrier following cerebral ischemia. J Neuropathol Exp Neurol. 1990;49:486-497.

21. ClinicalTrials.gov. Bevacizumab in reducing CNS side effects in patients who have undergone radiation therapy to the brain for primary brain tumor, meningioma, or head and neck cancer. Available at: http:// clinicaltrials.gov/ct2/results?term=NCT00492089. Accessed August 27, 2012.

22. ClinicalTrials.gov. A study of bevacizumab in combination with first- or second-line therapy in subjects with brain metastases due to non-squamous NSCLC (PASSPORT). Available at: http://clinicaltrials. gov/ct2/results?term=NCT00312728. Accessed August 27, 2012.
OncoTargets and Therapy

\section{Publish your work in this journal}

OncoTargets and Therapy is an international, peer-reviewed, open access journal focusing on the pathological basis of all cancers, potential targets for therapy and treatment protocols employed to improve the management of cancer patients. The journal also focuses on the impact of management programs and new therapeutic agents and protocols on

\section{Dovepress}

patient perspectives such as quality of life, adherence and satisfaction. The manuscript management system is completely online and includes a very quick and fair peer-review system, which is all easy to use. Visit http://www.dovepress.com/testimonials.php to read real quotes from published authors. 